\title{
Fluoropyrimidine plus cisplatin for patients with advanced or recurrent gastric cancer with peritoneal metastasis
}

\author{
Kohei Shitara $\cdot$ Ayako Mizota $\cdot$ Keitaro Matsuo • \\ Yozo Sato $\cdot$ Chihiro Kondo • Daisuke Takahari • \\ Takashi Ura $\cdot$ Masahiro Tajika $\cdot$ Kei Muro
}

Received: 4 November 2011/Accepted: 20 January 2012/Published online: 24 February 2012

(c) The International Gastric Cancer Association and The Japanese Gastric Cancer Association 2012

\begin{abstract}
Background There are few data on the efficacy of combination chemotherapy with a fluoropyrimidine plus cisplatin for patients with advanced or recurrent gastric cancer (AGC) complicated by peritoneal metastasis, especially massive ascites.

Methods We retrospectively evaluated the efficacy and safety of a fluoropyrimidine (S-1 or capecitabine) plus cisplatin as first-line chemotherapy in 120 patients with AGC and peritoneal metastasis.

Results Ascites was detected in 50 patients, with 11 patients having massive ascites. Median progression-free survival (PFS) and overall survival (OS) of all patients was 6.1 and 15.9 months, respectively. The PFS and OS were shorter in patients with massive ascites $(n=11 ; 3.7$ and 9.5 months) compared with patients with small or moderate ascites $(n=39 ; 5.8$ and 13.5 months) or patients without ascites ( $n=70 ; 6.9$ and 18.1 months). The objective response in terms of ascites was similar whether
\end{abstract}

K. Shitara $(\bowtie) \cdot$ A. Mizota - C. Kondo - D. Takahari - T. Ura · K. Muro

Department of Clinical Oncology, Aichi Cancer Center Hospital, 1-1 Kanokoden, Chikusa-ku, Nagoya, Aichi 464-8681, Japan e-mail: Kouheis0824@yahoo.co.jp

K. Matsuo

Division of Epidemiology and Prevention, Aichi Cancer Center Research Institute, Nagoya, Japan

Y. Sato

Department of Diagnostic and Interventional Radiology,

Aichi Cancer Center Hospital, Nagoya, Japan

M. Tajika

Department of Gastroenterology, Aichi Cancer Center Hospital, Nagoya, Japan ascites was massive (4 of 11 patients; $36.4 \%$ ) or small or moderate (16 of 39 patients; $41 \%$ ). The frequencies of grade 3 or higher toxicity or treatment discontinuation due to toxicity are relatively similar across ascites groups.

Conclusions Fluoropyrimidine plus cisplatin appears to be tolerated in selected patients with peritoneal metastasis.

Keywords Chemotherapy · Cisplatin · Fluoropyrimidine · Gastric cancer $\cdot$ Peritoneal metastasis

\section{Introduction}

Gastric cancer is the fourth most common malignancy in the world $(988,602$ cases in $2008,7.8 \%$ of all malignancies) and the second leading cause of cancer death (737,419 deaths, $9.7 \%$ of all cancer deaths) [1]. The prognosis for patients with advanced or recurrent gastric cancer (AGC) remains poor; chemotherapy confers only a minimal survival advantage, with a median overall survival (OS) of approximately 1 year. In a pivotal phase III trial (SPIRITS trial) in Japan that compared S-1 alone with S-1 plus cisplatin (combination $=\mathrm{SP}$ ), patients treated with SP showed a significantly higher response rate (54 vs. $31 \%$ ), longer progression-free survival (PFS; 6.0 vs. 4.0 months), and longer OS (13 vs. 11 months) than patients receiving $\mathrm{S}-1$ alone [2]. Therefore, SP is now considered to be one of the standard regimens for AGC in Japan. Capecitabine, another oral fluoropyrimidine, when combined with cisplatin (combination $=\mathrm{XP}$ ), is also reported to have an effectiveness that is statistically indistinguishable from that of 5-fluorouracil (5-FU) plus cisplatin (ML17032 trial [3]), which was used as a reference regimen in recent global studies, including those in Japan [4, 5]. Thus, the most commonly used treatments for AGC are combination 
chemotherapy regimens consisting of a fluoropyrimidine (5-FU or an oral fluoropyrimidine) plus a platinum agent, although docetaxel or anthracyclines are sometimes combined in Western countries [6, 7].

Peritoneal metastasis, a common type of metastasis in AGC, causes several complications such as ascites, bowel obstruction, and hydronephrosis_-all leading to a deterioration of the patient's general condition. Several reports have suggested that the presence of peritoneal metastasis or ascites is associated with poor survival in patients with AGC [8-11]. To improve the prognosis for patients with AGC and peritoneal metastasis, several clinical trials have been conducted [12-18]. However, there are few data on the efficacy of a fluoropyrimidine plus cisplatin for peritoneal metastasis as the current standard treatment for patients with AGC. Moreover, since patients with massive ascites have usually been excluded in previous pivotal randomized studies, the efficacy and feasibility in this patient population is also unclear. Therefore, we retrospectively evaluated the efficacy and safety of a fluoropyrimidine plus cisplatin regimen in patients with AGC and peritoneal metastasis.

\section{Patients and methods}

\section{Patients}

This retrospective study was designed to evaluate the efficacy and safety of first-line chemotherapy with a fluoropyrimidine plus cisplatin (SP and XP) in patients with AGC from January 2005 to March 2011. Since capecitabine was not available in Japan until February 2011, most patients had been treated by SP, although we included patients who had been treated with XP in the context of two global studies [3, 4]. Patients who had received XP plus experimental agents (i.e., trastuzumab or bevacizumab) were excluded from our analysis.

Eligibility criteria were as follows: (1) presence of histologically proven, inoperable AGC; (2) Eastern Cooperative Oncology Group performance status (ECOG PS) 0-2; (3) sufficient oral intake to take oral agents; (4) adequate bone marrow, hepatic, and renal function; (5) diagnosis of peritoneal metastasis, which could be confirmed either by macroscopic evaluation (upon laparotomy or laparoscopy) with cytology or by imaging data [computed tomography (CT) scan or barium enema] with relevant signs such as ascites, hydronephrosis, and intestinal stenosis; (6) no previous chemotherapy other than adjuvant chemotherapy, which was required to have been finished more than 6 months before enrollment. Written informed consent for chemotherapy was obtained from each patient prior to treatment initiation.
Treatment plan

Patients were treated with either: (1) a standard regimen of SP [S-1 $\left(80 \mathrm{mg} / \mathrm{m}^{2}\right)$ for 21 consecutive days followed by a 14-day rest; cisplatin $\left(60 \mathrm{mg} / \mathrm{m}^{2}\right)$ intravenous infusion on day 8] with repetition of the 35-day cycle [2]; or (2) XP [capecitabine $\left(1,000 \mathrm{mg} / \mathrm{m}^{2}\right)$ for 14 days followed by a 7-day rest; cisplatin $\left(80 \mathrm{mg} / \mathrm{m}^{2}\right)$ intravenous infusion on day 1] with repetition of the 21-day cycle [4, 5]. Intravenous hydration $(1,500 \mathrm{~mL})$ was performed on the day of cisplatin administration and on the next 2 days. Dose modification and scheduling of the two regimens were performed as reported in the literature [2, 4, 5]. Patients could continue with the fluoropyrimidine alone if they experienced severe toxicity with cisplatin. Treatment was discontinued if the tumor progressed, severe toxicity occurred, or at the patient's request.

Evaluation of treatment and statistical analysis

In patients with measurable lesions, the tumor response was assessed objectively according to the guidelines of the Response Evaluation Criteria In Solid Tumors (RECIST, ver. 1.0), and the best overall response was recorded as the antitumor effect for that patient. The objective response rate in these patients was presented as the percentage of patients with a complete response $(\mathrm{CR})$ or partial response (PR). According to the Japanese Classification of Gastric Carcinoma [19], the amount of ascites was assessed by a radiologist using CT. Response rate for ascites represented the percentage of patients with complete disappearance (CR) or a dramatic decrease in ascites (PR). Time to treatment failure (TTF) was measured from the date of initiation of chemotherapy to the date of the last administration of fluoropyrimidine or cisplatin. The PFS was measured from the date of chemotherapy to the date of progressive disease or death from any cause. The OS was estimated from the date of initiation of chemotherapy to the date of death or last follow-up visit. Median PFS and median OS were estimated by the Kaplan-Meier method. Toxicities were graded according to the National Cancer Institute's Common Terminology Criteria for Adverse Events, version 4.0.

Our primary interest was in comparing the clinical outcomes among patient groups that had different amounts of ascites. The amount of ascites was defined as follows: small (limited to pelvic cavity or around liver); moderate (not small or massive); or massive (continuous ascites from surface of liver to pelvic cavity). This definition of massive ascites was the same as that used in the JCOG 0106 study [13]. The volume of ascites was also estimated by the fivepoint method, as previously reported $[16,20]$. We divided patients into the following three groups: (1) patients 
without ascites; (2) patients with small or moderate ascites; and (3) patients with massive ascites.

$P$ values for testing differences in baseline characteristics and response rates of each ascites group were calculated for homogeneity using chi-square tests and for trends using Fisher's exact test. The PFS and OS were compared among the ascites groups by the log-rank test; the hazard ratio (HR) was calculated by the Cox proportional hazards model, and presented as HRs and $95 \%$ confidence intervals (95\% CIs). Statistical analyses were performed using STATA software (version 10; StataCorp LP, College Station, TX, USA). All tests were two sided, and $P<0.05$ was considered statistically significant.

\section{Results}

\section{Patient characteristics}

A total of 275 patients with AGC had received first-line chemotherapy with a fluoropyrimidine plus cisplatin regimen from January 2005 to March 2011. Of these patients, 120 patients met the inclusion criteria and were analyzed in this study. Patient characteristics are shown in Table 1. Most patients had PS 0 or 1; only 2 patients had PS 2 . Peritoneal metastasis was diagnosed by laparotomy or laparoscopy in 45 patients. The other 75 patients were diagnosed by imaging data including CT scan or barium enema. Ascites was detected in 50 patients $(42 \%)$ by CT scan: 27 patients $(23 \%)$ had small ascites; 12 patients (10\%) had moderate ascites; and 11 patients $(9 \%)$ had massive ascites. Of the patients with massive ascites, 5 patients underwent paracentesis prior to chemotherapy. The estimated volume of ascites according to this classification was as follows: median of $190 \mathrm{~mL}$ in small ascites (range, $<100-640 \mathrm{~mL}$ ); median of $990 \mathrm{~mL}$ in moderate ascites (range, $600-1,600 \mathrm{~mL}$ ); and median of 3,240 mL in massive ascites (range, 1,920-7,200 mL). The proportion of patients with lymph node metastasis or with two or more metastatic organs was higher in the patient group with small or moderate ascites than in the other two groups (Table $1, P=0.01$ ). Human epidermal growth factor receptor 2 (HER2) status was evaluated in 39 patients (22\%); four of these patients (10\%) were positive, which was defined as immunohistochemistry (IHC) $3+$ or IHC $2+$ plus amplification by fluorescence in situ hybridization (FISH). Of the 120 patients evaluated, 107 patients (89\%) had been treated with SP and 13 patients (11\%) with XP.

Treatment results and efficacy

The median TTF among all patients was 5.8 months, and cisplatin was administered a median of four times (range
0-13 times) during the median follow-up period of 34.9 months (Table 2). Three patients (2 patients without ascites and 1 patient with small ascites) started SP, but did not receive cisplatin on day 8 because of toxicity. After the initial dose, the dose of fluoropyrimidines was reduced in 23 patients (19\%) and the dose of cisplatin was reduced in 33 patients $(28 \%)$. One-hundred thirteen patients discontinued S-1 or capecitabine treatment for the following reasons: disease progression $(n=97 ; 81 \%)$, toxicity $(n=6 ; 5 \%)$, and other $(n=10 ; 8 \%)$.

The median numbers of times that cisplatin was administered within the ascites groups were as follows: 4 times in patients without ascites; 3 times in patients with small to moderate ascites; and 2 times in patients with massive ascites. The frequency of discontinuation due to toxicities and dose reduction was not higher in patients with massive ascites than in the other two groups (Table 2).

Of the 55 patients with measurable lesions, 23 patients achieved a CR $(n=1)$ or a PR $(n=22)$ for an overall response rate of $42.0 \%$ (95\% CI, 28.7-55.9\%; Table 3). Of the patients with ascites $(n=50)$, disappearance of ascites was observed in 8 patients (16\%), and a decrease of ascites was observed in 12 patients (24\%), for an overall response rate in terms of ascites of $40 \%$ (95\% CI, 26.4-54.8\%; Table 3). Response rates in terms of measurable lesions or ascites were relatively similar among the ascites groups (Table 3).

One hundred seven patients had already experienced disease progression at the time of analysis, with a median PFS of 6.1 months (95\% CI, 5.3-7.3 months) (Fig. 1). Eighty-four patients (70\%) were dead, with a median OS of 15.9 months (95\% CI, 12.8-18.4 months) (Fig. 1). Median PFS was shorter in patients with massive ascites (3.7 months; 95\% CI, 0.7-6.0 months) than in patients with small or moderate ascites (5.8 months; 95\% CI, 4.0-8.8 months; HR 0.45; 95\% CI, 0.22-0.93; $P=0.03$ ) or patients without ascites (6.9 months; 95\% CI, 5.5-9.0 months; HR 0.43; 95\% CI, 0.22-0.85; $P=0.02$ ) (Fig. 2). Median OS was also shorter in patients with massive ascites (9.5 months; 95\% CI, 0.5-not reached) than in patients with small or moderate ascites (13.5 months; 95\% CI, 9.4-17.0 months; HR 0.49; 95\% CI; $0.21-1.15 ; \quad P=0.1)$ or patients without ascites (18.1 months; 95\% CI, 14.5-20.0 months; HR 0.31; 95\% CI, 0.13-0.71; $P=0.006$ ) (Fig. 3).

Ninety-three patients $(78 \%)$ received second-line chemotherapy, most commonly $(n=69)$ with taxanes (paclitaxel or docetaxel). The proportion of patients having second-line chemotherapy was relatively similar among the ascites groups: 53 patients without ascites $(75.7 \%), 31$ patients with small to moderate ascites $(79.5 \%)$, and 9 patients with massive ascites (81.9\%). 
Table 1 Patient characteristics

\begin{tabular}{|c|c|c|c|c|}
\hline Characteristics & $\begin{array}{l}\text { All patients } \\
(n=120 \%)\end{array}$ & $\begin{array}{l}\text { Patients without } \\
\text { ascites }(n=70 \%)\end{array}$ & $\begin{array}{l}\text { Patients with small } \\
\text { to moderate ascites } \\
(n=39 \%)\end{array}$ & $\begin{array}{l}\text { Patients with } \\
\text { massive ascites } \\
(n=11 \%)\end{array}$ \\
\hline \multicolumn{5}{|l|}{ Age } \\
\hline Median (range) & $61(27-79)$ & $61(34-79)$ & $61(27-74)$ & $59(28-66)$ \\
\hline \multicolumn{5}{|l|}{ Gender } \\
\hline Male & $62(52)$ & $39(56)$ & $19(49)$ & $4(36)$ \\
\hline Female & $58(48)$ & $31(44)$ & $20(51)$ & $7(64)$ \\
\hline \multicolumn{5}{|l|}{ ECOG PS } \\
\hline 0 & $26(22)$ & $20(29)$ & $6(15)$ & $2(18)$ \\
\hline 1 & $92(77)$ & $50(71)$ & $31(79)$ & $9(82)$ \\
\hline 2 & $2(2)$ & 0 & $2(5)$ & 0 \\
\hline \multicolumn{5}{|l|}{ Histological type } \\
\hline Diffuse & $96(80)$ & $61(87)$ & $28(72)$ & $7(64)$ \\
\hline Intestinal & $24(20)$ & $9(13)$ & $11(28)$ & $4(36)$ \\
\hline \multicolumn{5}{|l|}{ Disease status } \\
\hline Advanced & $102(85)$ & $58(83)$ & $34(87)$ & $10(91)$ \\
\hline Recurrent & $18(15)$ & $12(17)$ & $5(13)$ & $1(9)$ \\
\hline \multicolumn{5}{|c|}{ Previous gastrectomy } \\
\hline No & $86(72)$ & $45(64)$ & $31(79)$ & $10(91)$ \\
\hline Yes & $34(28)$ & $25(36)$ & $8(21)$ & $1(9)$ \\
\hline \multicolumn{5}{|c|}{ Prior adjuvant chemotherapy } \\
\hline No & $110(92)$ & $62(89)$ & $37(95)$ & $11(100)$ \\
\hline Yes & $10(8)$ & $8(11)$ & $2(5)$ & 0 \\
\hline \multicolumn{5}{|l|}{ Site of metastasis } \\
\hline Lymph node & $48(40)$ & $22(31)$ & $23(59)$ & $3(27)$ \\
\hline Liver & $11(9)$ & $4(6)$ & $6(15)$ & $1(9)$ \\
\hline Ovary & $11(9)$ & $4(6)$ & $5(13)$ & $2(18)$ \\
\hline \multicolumn{5}{|c|}{ Number of metastatic organs } \\
\hline 1 & $56(47)$ & $41(59)$ & $10(26)$ & $5(45)$ \\
\hline 2 or more & $64(53)$ & $29(41)$ & $29(74)$ & $6(55)$ \\
\hline
\end{tabular}

\section{Toxicity}

Toxicity is shown in Table 4. The frequencies of any grade 3-4 hematological toxicity were $27 \%$ (19 of 70 patients) in patients without ascites, $41 \%$ (16 of 39 patients) in patients with small to moderate ascites, and $27 \%$ (3 of 11 patients) in patients with massive ascites; the frequency in patients with massive ascites was not significantly higher. The frequencies of any grade 3-4 nonhematological toxicity also did not differ significantly among patients without ascites $(34 \% ; n=24)$, patients with small or moderate ascites $(26 \% ; n=10)$, or patients with massive ascites $(45 \% ; n=5)$. The frequency of grade 3 or higher anorexia tended to be higher in patients with massive ascites $(36 \% ; n=4)$ than in patients without ascites $(19 \% ; n=13)$ or patients with small or moderate ascites $(15 \% ; n=6)$. No patients experienced grade 3 or higher renal toxicity.

\section{Discussion}

We retrospectively evaluated the efficacy and safety of a fluoropyrimidine plus cisplatin regimen for patients with AGC and peritoneal metastasis. Median PFS and OS were similar to that of the SPIRITS trial, in which about $30 \%$ of patients had peritoneal metastasis (34\% in SP group, $24 \%$ in S-1 group) [2]. The frequencies of common toxicities in our analysis were also compatible with that in the SPIRITS trial; therefore, a fluoropyrimidine (S-1 or capecitabine) plus cisplatin regimen is considered to be effective and feasible for treatment of patients with peritoneal metastasis.

In our analysis, PFS and OS were worse in patients with massive ascites than in patients without ascites or patients with small or moderate ascites. Although the incidence of anorexia was higher in patients with massive ascites, the frequencies of discontinuation or dose reduction due to 
Table 2 Treatment results

\begin{tabular}{|c|c|c|c|c|}
\hline Variables & $\begin{array}{l}\text { All patients } \\
(n=120 \%)\end{array}$ & $\begin{array}{l}\text { Patients without } \\
\text { ascites }(n=70 \%)\end{array}$ & $\begin{array}{l}\text { Patients with small } \\
\text { or moderate ascites } \\
(n=39 \%)\end{array}$ & $\begin{array}{l}\text { Patients with } \\
\text { massive ascites } \\
(n=11 \%)\end{array}$ \\
\hline \multicolumn{5}{|l|}{ Median TTF } \\
\hline Median (months, range) & $5.8(0.3-33.8)$ & $6.5(0.3-33.8)$ & $5.7(0.3-28.4)$ & $3.4(0.4-10.6)$ \\
\hline \multicolumn{5}{|l|}{ Cisplatin administration } \\
\hline Median number of times & $4(0-13)$ & $4(0-13)$ & $3(0-12)$ & $2(1-6)$ \\
\hline \multicolumn{5}{|c|}{ Dose reduction in fluoropyrimidine } \\
\hline Yes & $23(19)$ & 13 (19) & $10(26)$ & $0(0)$ \\
\hline \multicolumn{5}{|l|}{ Dose reduction in cisplatin } \\
\hline Yes & $33(28)$ & $23(33)$ & $10(26)$ & $0(0)$ \\
\hline \multicolumn{5}{|c|}{ Cause of discontinuation of cisplatin } \\
\hline Progressive disease & $52(43)$ & $27(39)$ & $17(44)$ & $8(73)$ \\
\hline Toxicities & $34(28)$ & $22(31)$ & $9(23)$ & $3(27)$ \\
\hline Other & $31(26)$ & $18(26)$ & $13(33)$ & $0(0)$ \\
\hline Ongoing & $3(3)$ & $3(4)$ & 0 & 0 \\
\hline \multicolumn{5}{|c|}{ Cause of S-1 or capecitabine discontinuation } \\
\hline Progressive disease & $97(81)$ & $52(74)$ & $35(90)$ & $10(91)$ \\
\hline Toxicities & $6(5)$ & $4(6)$ & $2(5)$ & $0(0)$ \\
\hline Other & $10(8)$ & $9(13)$ & $1(3)$ & 0 \\
\hline Ongoing & $7(6)$ & $5(4)$ & $1(3)$ & $1(9)$ \\
\hline
\end{tabular}

$T T F$ time to treatment failure

Table 3 Objective response rates in measurable lesions and ascites

\begin{tabular}{|c|c|c|c|c|c|c|c|c|c|}
\hline Groups & $N$ & $\mathrm{CR}$ & PR & SD & PD & $\mathrm{NE}$ & ORR $(\%)$ & $95 \%$ CI $(\%)$ & $P$ value \\
\hline All patient with target lesions & 55 & 1 & 22 & 23 & 5 & 4 & 42.0 & $28.7-55.9$ & 0.87 \\
\hline No ascites & 25 & 1 & 10 & 10 & 0 & 4 & 44.0 & $24.4-65.1$ & \\
\hline Small to moderate ascites & 26 & 0 & 10 & 12 & 4 & 0 & 38.5 & $20.2-59.4$ & \\
\hline Massive ascites & 4 & 0 & 2 & 1 & 1 & 0 & 50.0 & $6.8-93.2$ & \\
\hline All patient with ascites & 50 & 8 & 12 & 17 & 10 & 3 & 40.0 & $26.4-54.8$ & 0.78 \\
\hline Small to moderate ascites & 39 & 8 & 8 & 14 & 6 & 3 & 41.0 & $25.6-57.9$ & \\
\hline Massive ascites & 11 & 0 & 4 & 3 & 4 & 0 & 36.4 & $10.9-69.2$ & \\
\hline
\end{tabular}

$C R$ complete response, $P R$ partial response, $S D$ stable disease, $P D$ progressive disease, $N E$ not evaluable, $O R R$ objective response rate, $C I$ confidence interval

a Comparison of ORR between 3 groups

toxicity were not higher. Therefore, this treatment may be feasible even for patients with massive ascites if they have good performance status, sufficient oral intake, and adequate organ function. However, median treatment duration and PFS are quite short in patients with massive ascites compared with other patients; therefore, more effective treatments may be necessary to improve the poor prognosis.

To date, several clinical trials have been conducted or are ongoing in patients with peritoneal metastasis. The JCOG 9603 trial showed the efficacy of 5-FU plus methotrexate in patients with AGC with ascites: a response rate in terms of ascites of $35.1 \%$ was noted [12]. The JCOG 0106 study was conducted to compare infused 5-FU versus
5-FU plus methotrexate in patients with AGC and peritoneal metastasis, but it did not show a superiority of 5-FU plus methotrexate [13]. Although the JCOG 0106 trial did not include patients with massive ascites and did not evaluate response in terms of ascites, improvement of oral intake was reported in $48 \%$ of patients who were unable to eat at the study outset [13]; this finding suggests substantial efficacy of the 5-FU-based therapy in patients with AGC and peritoneal metastasis.

In the SPIRITS trial, combination treatment with cisplatin (SP) showed favorable results compared with S-1 alone in the subset of patients with peritoneal metastasis [2]. Although patients with massive ascites were excluded and detailed information about ascites is not available in 


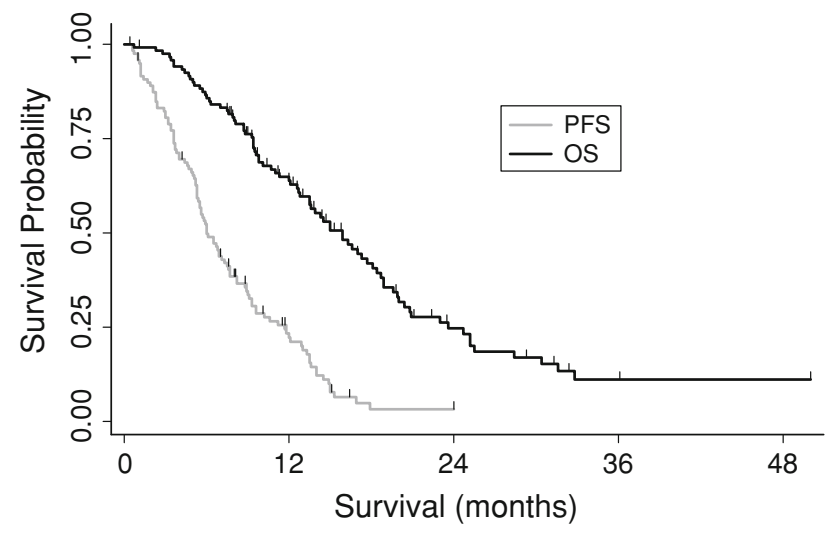

Fig. 1 Progression-free survival and overall survival. Median PFS was 6.1 months (95\% CI, 5.3-7.3 months), and median OS was 15.9 months (95\% CI, 12.8-18.4 months)

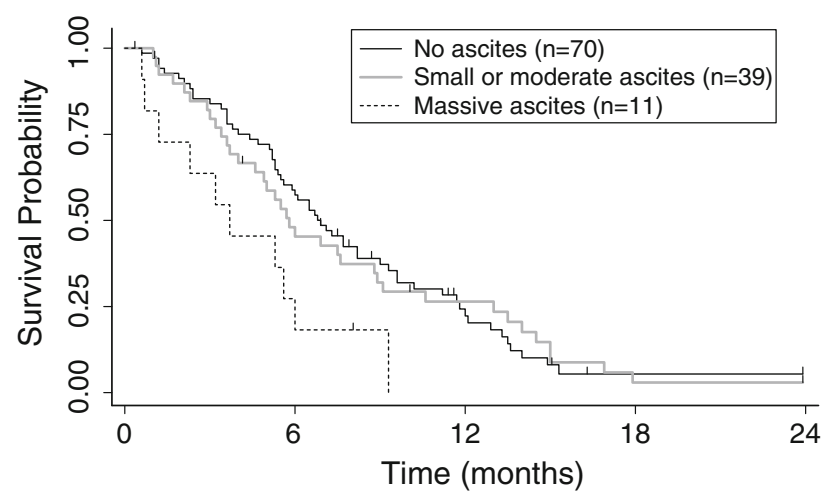

Fig. 2 Progression-free survival by ascites group. Median PFS was shorter in patients with massive ascites (3.7 months; 95\% CI, 0.7-6.0 months) than in patients with small or moderate ascites (5.8 months; 95\% CI, 4.0-8.8 months; HR 0.45; 95\% CI, 0.22-0.93; $P=0.03)$ or patients without ascites (6.9 months; 95\% CI, 5.5-9.0 months; HR 0.43; 95\% CI, $0.22-0.85 ; P=0.02$ )

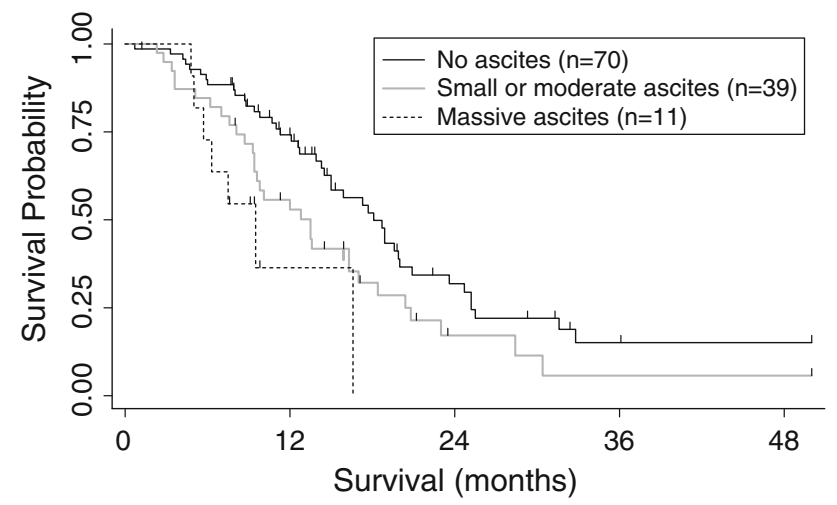

Fig. 3 Overall survival according to ascites group. Median OS was shorter in patients with massive ascites (9.5 months; 95\% CI, 0.5-not reached) than in patients with small or moderate ascites (13.5 months; 95\% CI, 9.4-17.0 months; HR 0.49; 95\% CI, $0.21-1.15 ; P=0.1)$ or patients without ascites (18.1 months; 95\% CI, 14.5-20.0 months; HR $0.31 ; 95 \%$ CI, $0.13-0.71 ; P=0.006$ ) the SPIRITS trial, this result suggests that cisplatin is also an important agent for patients with peritoneal metastasis. Oxaliplatin, another platinum agent, showed noninferior efficacy with significantly less renal toxicity [7] and gastrointestinal toxicity [21] in comparison with cisplatin. A 5-FU and oxaliplatin regimen was also evaluated in patients with AGC and ascites, with a response rate in terms of ascites of $33 \%$ with low toxicities [14].

Another effective drug type for patients with peritoneal metastasis is a taxane agent (paclitaxel or docetaxel). The JCOG 0407 trial is a randomized phase II study that compared second-line chemotherapy of weekly paclitaxel with 5-FU-based chemotherapy for patients with AGC and peritoneal metastasis [15]. The efficacy of paclitaxel was suggested by a longer PFS in the paclitaxel arm [15]. A phase II study of weekly paclitaxel for patients with malignant ascites, which included mostly patients with massive ascites (median 2,796 mL), showed a decrease in ascites and improvement of performance status in $39.1 \%$ of patients [16]. Combination treatment with 5-FU and paclitaxel also showed a high response rate $(44 \%)$ in patients with massive ascites [17]. These results suggest the apparent efficacy of paclitaxel in patients with AGC and ascites. In our study, second-line chemotherapy, mainly with taxanes, was used in most patients, including those with massive ascites-possibly contributing to the relatively long survival after first-line chemotherapy. Additionally, a recent phase II study that evaluated S-1 combined with intravenous and intraperitoneal chemotherapy with paclitaxel included 40 patients with peritoneal metastasis in whom overall survival was as impressively long as 22.5 months [18]. Also, in the 30 patients with ascites in that study, the response in terms of ascites was reported to be as high as $60 \%$ [18]. These results compare favorably with those from our analysis. The efficacy of intraperitoneal administration of paclitaxel was suggested in a randomized study of patients with ovarian cancer and peritoneal metastasis [22]. Therefore, this treatment may be promising in AGC, especially for patients with peritoneal metastasis. Currently, a randomized study comparing S-1 plus intraperitoneal and intravenous paclitaxel versus S-1 plus cisplatin is ongoing.

It is important to note the limitations of the present study. First, it was a retrospective analysis in a single institution with patients that had sufficient oral intake and adequate organ function. None of the patients had symptoms or complications such as decreased oral intake or renal dysfunction due to hydronephrosis; the treatment regimen used in our study may not be feasible for such patients. Specifically, patients with peritoneal metastasis frequently have an inability to eat [23], making it impossible to use oral agents in such patients, and patients with renal dysfunction should not be given cisplatin. Therefore, 
Table 4 Toxicities

\begin{tabular}{|c|c|c|c|c|c|c|c|c|c|}
\hline & \multicolumn{2}{|c|}{ All $(n=120 \%)$} & \multicolumn{2}{|c|}{$\begin{array}{l}\text { Patients without ascites } \\
(n=70 \%)\end{array}$} & \multicolumn{2}{|c|}{$\begin{array}{l}\text { Patients with small or } \\
\text { moderate ascites }(n=39 \%)\end{array}$} & \multicolumn{2}{|c|}{$\begin{array}{l}\text { Patients with massive } \\
\text { ascites }(n=11 \%)\end{array}$} & \multirow[t]{2}{*}{$P$ value $^{\mathrm{a}}$} \\
\hline & All (\%) & G3-4 (\%) & All (\%) & G3-4 (\%) & All (\%) & G3-4 (\%) & All (\%) & G3-4 (\%) & \\
\hline \multicolumn{10}{|l|}{ Hematological toxicity } \\
\hline Any & $75(62)$ & $38(32)$ & $40(57)$ & $19(27)$ & $27(69)$ & $16(41)$ & $8(73)$ & $3(27)$ & 0.31 \\
\hline Leukopenia & $58(48)$ & $15(12)$ & $29(41)$ & $9(13)$ & $22(56)$ & $5(13)$ & $7(64)$ & $1(9)$ & 0.94 \\
\hline Neutropenia & $60(50)$ & $28(23)$ & $31(44)$ & $16(23)$ & $22(56)$ & $10(26)$ & $7(64)$ & $2(18)$ & 0.89 \\
\hline Anemia & $51(42)$ & $12(10)$ & 27 (39) & $6(9)$ & $19(49)$ & $5(13)$ & $5(46)$ & $1(9)$ & 0.77 \\
\hline Thrombocytopenia & $25(21)$ & $4(3)$ & $14(20)$ & $3(4)$ & $9(23)$ & $1(3)$ & $2(18)$ & 0 & 0.72 \\
\hline \multicolumn{10}{|c|}{ Nonhematological toxicity } \\
\hline Any & $96(80)$ & $39(33)$ & $59(84)$ & $24(34)$ & $29(74)$ & $10(26)$ & $8(73)$ & $5(45)$ & 0.45 \\
\hline Nausea & $73(61)$ & $17(14)$ & $44(63)$ & $12(17)$ & $22(56)$ & $5(13)$ & $7(64)$ & $2(18)$ & 0.71 \\
\hline Vomiting & $30(25)$ & $4(3)$ & $18(26)$ & $3(4)$ & 7 (18) & $0(0)$ & $5(45)$ & $1(9)$ & 0.26 \\
\hline Anorexia & $80(67)$ & 23 (19) & $45(64)$ & $13(19)$ & $28(72)$ & $6(15)$ & $7(64)$ & $4(36)$ & 0.29 \\
\hline Fatigue & $55(46)$ & $8(7)$ & $32(46)$ & $6(9)$ & $19(49)$ & $2(5)$ & $4(36)$ & $1(9)$ & 0.51 \\
\hline Diarrhea & $25(20)$ & $5(4)$ & $18(26)$ & $4(6)$ & $5(13)$ & $1(3)$ & $2(18)$ & 0 & 0.56 \\
\hline Increased creatinine & $17(14)$ & 0 & 13 (19) & 0 & $4(10)$ & 0 & $1(9)$ & 0 & $0.43^{\mathrm{b}}$ \\
\hline Stomatitis & $17(14)$ & $2(2)$ & $11(16)$ & $2(3)$ & $4(10)$ & 0 & $2(18)$ & 0 & 0.48 \\
\hline Rash & $4(3)$ & 0 & $3(4)$ & 0 & $1(3)$ & 0 & 0 & 0 & $0.78^{\mathrm{b}}$ \\
\hline Hand-foot syndrome & $9(8)$ & 0 & $5(7)$ & 0 & $4(10)$ & 0 & 0 & 0 & $0.69^{\mathrm{b}}$ \\
\hline Febrile neutropenia & $2(2)$ & $2(2)$ & 0 & $2(3)$ & 0 & 0 & 0 & 0 & 0.48 \\
\hline
\end{tabular}

${ }^{a}$ Comparison in grade 3 or more

b Comparison in all grades

in these types of patients, other treatments such as intravenous 5-FU or combination therapy with taxanes may be the preferred choice. Second, we included both SP and XP in this study, although most patients were treated with SP. Direct comparison of S-1 and capecitabine as well as indirect comparisons of several randomized studies using SP and XP suggest that these two treatments have similar efficacies $[2,3,24]$. Additionally, our retrospective analysis comparing these two treatment regimens showed that they have similar efficacies and safeties [25]. S-1 was suggested to be more efficacious than 5-FU in patients with diffuse-type AGC [26] or AGC associated with high dihydropyrimidine dehydrogenase (DPD), with diffusetype tumors being more commonly associated with high DPD than intestinal-type tumors are [27]. Since diffusetype cases are commonly associated with peritoneal metastasis, S-1 may be preferable for the treatment of AGC in this setting. In contrast, several small analyses have suggested that capecitabine is effective at treating highthymidine phosphorylase (TP) gastric cancer [28, 29]; for such tumors, 5-FU and S-1 are reported to be relatively ineffective compared with their efficacy towards low-TP gastric cancer [30, 31]. The exact impact of using biomarkers or histology to select among 5-FU, S-1, and capecitabine should be evaluated in ongoing randomized studies.
In conclusion, although our findings are limited by the retrospective study design and small number of patients, a regimen consisting of a fluoropyrimidine plus cisplatin appears to be tolerated in selected patients with peritoneal metastasis.

Acknowledgments The manuscript has not been published nor submitted for publication elsewhere, except as a brief abstract in the proceedings of a scientific meeting or symposium (two topics were presented at the 49th Annual Meeting of Japanese Society of Clinical Oncology, October 27-29, 2011).

Conflict of interest None.

\section{References}

1. International Agency for Research on Cancer; GLOBOCAN 2008. http://www-dep.iarc.fr/CancerMondial.htm.

2. Koizumi W, Narahara H, Hara T, Takagane A, Akiya T, Takagi $\mathrm{M}$, et al. S-1 plus cisplatin versus S-1 alone for first-line treatment of advanced gastric cancer (SPIRITS trial): a phase III trial. Lancet Oncol. 2008;9:215-21.

3. Kang YK, Kang WK, Shin DB, Chen J, Xiong J, Wang J, et al. Capecitabine/cisplatin versus 5 -fluorouracil/cisplatin as first-line therapy in patients with advanced gastric cancer: a randomised phase III noninferiority trial. Ann Oncol. 2009;20:666-73.

4. Bang YJ, Van Cutsem E, Feyereislova A, Chung HC, Shen L, Sawaki A, et al. Trastuzumab in combination with chemotherapy 
versus chemotherapy alone for treatment of HER2-positive advanced gastric or gastro-oesophageal junction cancer (ToGA): a phase 3, open-label, randomised controlled trial. Lancet. 2010; 376:687-97.

5. Ohtsu A, Shah MA, Van Cutsem E, Rha SY, Sawaki A, Park SR, et al. Bevacizumab in combination with chemotherapy as firstline therapy in advanced gastric cancer: a randomized, doubleblind, placebo-controlled phase III study. J Clin Oncol. 2011. Epub ahead of print.

6. Van Cutsem E, Moiseyenko VM, Tjulandin S, Majlis A, Constenla M, Boni C, et al. Phase III study of docetaxel and cisplatin plus fluorouracil compared with cisplatin and fluorouracil as firstline therapy for advanced gastric cancer: a report of the V325 Study Group. J Clin Oncol. 2006;24:4991-7.

7. Cunningham D, Starling N, Rao S, Iveson T, Nicolson M, Coxon F, et al. Capecitabine and oxaliplatin for advanced esophagogastric cancer. N Engl J Med. 2008;358:36-46.

8. Chau I, Norman AR, Cunningham D, Waters JS, Oates J, Ross PJ. Multivariate prognostic factor analysis in locally advanced and metastatic esophago-gastric cancer-pooled analysis from three multicenter, randomized, controlled trials using individual patient data. J Clin Oncol. 2004;22:2395-403.

9. Lee J, Lim T, Uhm J, Park K, Park S, Lee S, et al. Prognostic model to predict survival following first-line chemotherapy in patients with metastatic gastric adenocarcinoma. Ann Oncol. 2007;18:886-91.

10. Kim JG, Ryoo BY, Park YH, Kim BS, Kim TY, Im YH, et al. Prognostic factors for survival of patients with advanced gastric cancer treated with cisplatin-based chemotherapy. Cancer Chemother Pharmacol. 2008;61:301-7.

11. Koo DH, Ryoo BY, Kim HJ, Ryu MH, Lee SS, Moon JH, et al. A prognostic model in patients who receive chemotherapy for metastatic or recurrent gastric cancer: validation and comparison with previous models. Cancer Chemother Pharm. 2011;68:913-21.

12. Yamao T, Shimada Y, Shirao K, Ohtsu A, Ikeda N, Hyodo I, et al. Phase II study of sequential methotrexate and 5-fluorouracil chemotherapy against peritoneally disseminated gastric cancer with malignant ascites: a report from the Gastrointestinal Oncology Study Group of the Japan Clinical Oncology Group, JCOG 9603 Trial. Jpn J Clin Oncol. 2004;34:316-22.

13. Shirao K, Boku N, Yamada Y, Yamaguchi K, Doi T, Takiuchi H, et al. Randomized phase III study of 5-fluorouracil continuous infusion (5FUci) versus methotrexate and 5-FU sequential therapy (MF) in gastric cancer with peritoneal metastasis (JCOG0106). J Clin Oncol. 2009;27:15s (suppl; abstr 4545).

14. Oh SY, Kwon HC, Lee S, Lee DM, Yoo HS, Kim SH, et al. A Phase II study of oxaliplatin with low-dose leucovorin and bolus and continuous infusion 5-fluorouracil (modified FOLFOX4) for gastric cancer patients with malignant ascites. Jpn J Clin Oncol. 2007;37:930-5.

15. Takiuchi H, Fukuda H, Boku N, Shimada Y, Nasu J, Hamamoto Y, et al. Randomized phase II study of best-available 5-fluorouracil (5$\mathrm{FU})$ versus weekly paclitaxel in gastric cancer (GC) with peritoneal metastasis (PM) refractory to 5-FU-containing regimens (JCOG0407). J Clin Oncol. 2010;28:15s (suppl; abstr 4052).

16. Imamoto H, Oba K, Sakamoto J, Iishi H, Narahara H, Yumiba T, et al. Assessing clinical benefit response in the treatment of gastric malignant ascites with non-measurable lesions: a multicenter phase II trial of paclitaxel for malignant ascites secondary to advanced/ recurrent gastric cancer. Gastric Cancer. 2011;14:81-90.

17. Takiuchi H, Yasui $H$, Nishina $T$, Takahari $D$, Nakayama $N$, Tairan K, et al. Multicenter feasibility study of 5-FU, leucovorin, plus paclitaxel (FLTAX) for peritoneal disseminated gastric cancer with massive ascites or inadequate oral intake. J Clin Oncol. 2011;29 (suppl 4; abstr 119).
18. Ishigami H, Kitayama J, Kaisaki S, Hidemura A, Kato M, Otani $\mathrm{K}$, et al. Phase II study of weekly intravenous and intraperitoneal paclitaxel combined with S-1 for advanced gastric cancer with peritoneal metastasis. Ann Oncol. 2010;21:67-70.

19. Japanese Gastric Cancer Association. Japanese classification of gastric carcinoma (2nd English edition) - response assessment of chemotherapy and radiotherapy for gastric carcinoma: clinical criteria. Gastric Cancer. 2001;4:1-8.

20. Oriuchi N, Nakajima T, Mochiki E, Takeyoshi I, Kanuma T, Endo $\mathrm{K}$, et al. A new, accurate and conventional five-point method for quantitative evaluation of ascites using plain computed tomography in cancer patients. Jpn J Clin Oncol. 2005; 35:386-90.

21. Al-Batran SE, Hartmann JT, Probst S, Schmalenberg H, Hollerbach S, Hofheinz R, et al. Phase III trial in metastatic gastroesophageal adenocarcinoma with fluorouracil, leucovorin plus either oxaliplatin or cisplatin: a study of the Arbeitsgemeinschaft Internistische Onkologie. J Clin Oncol. 2008;26:1435-42.

22. Armstrong DK, Bundy B, Wenzel L, Huang HQ, Baergen R, Lele $\mathrm{S}$, et al. Intraperitoneal cisplatin and paclitaxel in ovarian cancer. N Engl J Med. 2006;354:34-43.

23. Shitara K, Ito S, Sawaki A, Tajika M, Kawai H, Yokota T, et al. Improvement of oral intake following chemotherapy in gastric cancer patients with an inability to eat. Oncology. 2010;79: 211-8.

24. Lee JL, Kang YK, Kang HJ, Lee KH, Zang DY, Ryoo BY, et al. A randomised multicentre phase II trial of capecitabine vs S-1 as first-line treatment in elderly patients with metastatic or recurrent unresectable gastric cancer. Br J Cancer. 2008;99:584-90.

25. Shitara K, Sawaki A, Matsuo K, Kondo C, Takahari D, Ura T, et al. A retrospective comparison of S-1 plus cisplatin and capecitabine plus cisplatin for patients with advanced or recurrent gastric cancer. 49th Annual Meeting of Japanese Society of Clinical Oncology, October 27-29, 2011: Oral session 6-5.

26. Ajani JA, Rodriguez W, Bodoky G, Moiseyenko V, Lichinitser $\mathrm{M}$, Gorbunova V, et al. Multicenter phase III comparison of cisplatin/S-1 with cisplatin/infusional fluorouracil in advanced gastric or gastroesophageal adenocarcinoma study: the FLAGS trial. J Clin Oncol. 2010;28:1547-53.

27. Yamada Y, Yamamoto S, Ohtsu A, Suzuki Y, Nasu J, Yamaguchi $\mathrm{K}$, et al. Impact of dihydropyrimidine dehydrogenase status of biopsy specimens on efficacy of irinotecan plus cisplatin, S-1, or 5-FU as first-line treatment of advanced gastric cancer patients in JCOG9912. J Clin Oncol. 2009;27:15s (suppl; abstr 4535).

28. Koizumi W, Saigenji K, Nakamaru N, Okayasu I, Kurihara M. Prediction of response to $5^{\prime}$-deoxy-5-fluorouridine ( $5^{\prime}$-DFUR) in patients with inoperable advanced gastric cancer by immunostaining of thymidine phosphorylase/platelet-derived endothelial cell growth factor. Oncology. 1999;56:215-22.

29. Takiguchi N, Ishii R, Koda K, Oda K, Miyazaki M. Thymidine phosphorylase expression correlates with malignant potential and anti-tumor effect of doxifluridine on gastric cancer: multivariate analysis for adjuvant chemotherapy doxifluridine vs 5-fluorouracil. Oncol Rep. 2003;10:1105-11.

30. Ichikawa W, Takahashi T, Suto K, Hirayama R. Gene expressions for thymidylate synthase (TS), orotate phosphoribosyltransferase (OPRT), and thymidine phosphorylase (TP), not dihydropyrimidine dehydrogenase (DPD), influence outcome of patients (pts) treated with S-1 for gastric cancer (GC). J Clin Oncol Meet Abstr. 2004;22:4050.

31. Napieralski R, Ott K, Kremer M, Specht K, Vogelsang H, Becker $\mathrm{K}$, et al. Combined GADD45A and thymidine phosphorylase expression levels predict response and survival of neoadjuvant treated gastric cancer patients. Clin Cancer Res. 2005;11: $3025-31$. 